\title{
Percepciones de maestros y estudiantes sobre el uso del triplete químico en los procesos de enseñanza-aprendizaje
}

\author{
Teachers' and Students' Perceptions on the use of the chemical triplet in the teaching- \\ learning processes
}

\section{Percepções de professores e alunos no uso do trigêmeo químico nos processos de ensino-aprendizagem}

\author{
Danny José Lorduy ${ }^{10}$ \\ Claudia Patricia Naranjo ${ }^{(\mathbb{D}}$
}

Recibido: abril 2020

Aceptado: agosto 2020

Para citar este artículo: Lorduy, D. J., Naranjo, C. P. (2020). Percepciones de maestros y estudiantes sobre el uso del triplete químico en los procesos de enseñanza-aprendizaje. Revista Científica, 39(3), 324-340. https://doi. org/10.14483/23448350.16427

\section{Resumen}

El objetivo de este artículo fue indagar las percepciones de maestros y estudiantes de Ciencias sobre nociones científicas, dominios u obstáculos acerca del uso del triplete químico y saltos entre los niveles representacionales, durante los procesos de enseñanza y aprendizaje de la asignatura de Química. Se utilizó una metodología cualitativa con enfoque fenomenológico. La indagación se llevó a cabo durante ocho semanas del año 2019, por medio de tres grupos de discusión de nueve estudiantes y entrevistas a profundidad a siete maestros de Ciencias. Se realizó un análisis de contenido en torno a dos categorías centrales de análisis: representaciones en Química y enseñanza y aprendizaje de la Química. Se encontró que los maestros de Ciencias no poseen un dominio acerca del uso del triplete químico y saltos entre los niveles representacionales, lo que genera obstáculos para los procesos de enseñanza y aprendizaje de la química. Las respuestas dadas por los estudiantes se ubicaron en tres categorías centrales: nociones químicas, qué química aprender y para qué aprender química. Se usaron los niveles macro y simbólico para comprender y explicar fenómenos químicos del contexto relacionados con aquellas características que perciben. De este estudio se puede concluir que las percepciones de los estudiantes se centraron en interpretar el fenómeno en términos de la formación de nuevas sustancias, utilizando un lenguaje impreciso e indiferenciado para expresar sus nociones frente los conceptos de Química. Además, se recomienda que el maestro abandone el enfoque de transmisión de conocimientos y tome consciencia sobre la contextualización de fenómenos a partir del triplete químico.

Palabras clave: educación química contextualizada, enseñanza y aprendizaje de la Química, percepciones de maestros y estudiantes, triplete químico.

1. Universidad de Córdoba, Montería, Córdoba, Colombia. dlorduyflorez@correo.unicordoba.edu.co

2. Universidad de Córdoba, Montería, Córdoba, Colombia. cpnaranjo@correo.unicordoba.edu.co 


\section{Abstract}

The objective of this article was to inquire into the perceptions of science teachers and students, about scientific notions, domains or obstacles about the use of the chemical triplet and jumps between the representative levels, during the chemistry teaching and learning processes. A qualitative methodology with a phenomenological approach was used. The investigation was carried out during eight weeks of the year 2019, through discussion groups to three groups of nine students and in-depth interviews with seven science teachers. A content analysis was conducted around two central categories of analysis: representations in chemistry and chemistry teaching and learning. It was found that science teachers do not have a command of the use of the chemical triplet and jumps between the representational levels, creating obstacles for the chemistry teaching and learning processes. The answers given by the students were located in three central categories: chemical notions, what chemistry to learn and why to learn chemistry. The use of the macro and symbolic level was found to understand and explain chemical phenomena of the context, related to those characteristics that they perceive. This study allows us to conclude that the students' perceptions focused on interpreting the phenomenon in terms of the formation of new substances, using imprecise and undifferentiated language to express their notions regarding chemical concepts. Furthermore, the teacher must abandon the knowledge transmission approach and become aware of the contextualization of phenomena from the chemical triplet.

Keywords: contextualized chemistry education, chemistry teaching and learning, teacher and student perceptions, chemical triplet.

\section{Resumo}

O objetivo deste artigo foi investigar as percepções dos professores e alunos de ciências, sobre noções científicas, domínios ou obstáculos sobre o uso do tripleto químico e saltos entre os níveis representacionais, durante os processos de ensino e aprendizagem de química. Foi utilizada uma metodologia qualitativa com abordagem fenomenológica. A investigação foi realizada durante oito semanas do ano de 2019, por meio de grupos de discussão para três grupos de nove alunos e entrevistas em profundidade com sete professores de ciências. Foi realizada uma análise de conteúdo em torno de duas categorias centrais de análise: representações em química e ensino e aprendizagem de química. Verificou-se que os professores de ciências não dominam o uso do trigêmeo químico e salta entre os níveis representacionais, criando obstáculos para os processos de ensino e aprendizagem de química. As respostas dadas pelos alunos estavam localizadas em três categorias centrais: noções químicas, que química aprender e por que aprender química. Verificou-se que o uso do nível macro e simbólico compreende e explica fenômenos químicos do contexto, relacionados às características que eles percebem. Este estudo permite concluir que as percepções dos alunos se concentraram na interpretação do fenômeno em termos de formação de novas substâncias, utilizando linguagem imprecisa e indiferenciada para expressar suas noções sobre conceitos químicos. Além disso, o professor deve abandonar a abordagem de transmissão de conhecimento e tomar consciência da contextualização dos fenômenos a partir do trigêmeo químico.

Palavras-chaves: educação química contextualizada, ensino e aprendizagem de química, percepções de professores e alunos, trigêmeo químico.

\section{Introducción}

La educación en Ciencias parte de tres componentes básicos, dominios o niveles, los cuales son utilizados particularmente en Química y fueron propuestos por Alex. H. Johnstone (1982, 1991, 1993, 2000) en un modelo triangular. Cada vértice se encuentra etiquetado de la siguiente manera: 1) macroscópico: que pertenece a todo lo observable, tangible o concreto; 2) submicroscópico: que referencia a lo molecular, cinético y átomos; y 3) simbólico: a lo que Johnstone definió Representational Chemistry, donde se incluyen los símbolos, la estequiometría, ecuaciones y las matemáticas (Cutrera y Stipcich, 2016). Por tanto, se deben proveer alternativas didácticas genuinas que empleen en los procesos de enseñanza y aprendizaje el 
triplete químico (Taber, 2019). Desde el punto de vista del aprendizaje de la Química se debe propiciar el desarrollo de procesos comprensivos con una perspectiva holística. Por ello, la enseñanza debe estar inmersa en una profundización a través de los vértices del triplete Químico (Caamaño, 2011, 2014).

Por otro lado, la educación química contextualizada promueve aprendizajes profundos en los estudiantes, mediante la comprensión objetiva y crítica de los fenómenos naturales (Meroni et al., 2015). Por lo anterior, las metodologías didácticas contextualizadas generan actitudes positivas y disposición en los estudiantes, lo que permite que perciban el conocimiento científico como parte de su cotidianidad $y$, asimismo, reflexionar sobre los distintos factores que pueden afectar su contexto (Caamaño, 2018; Meroni et al., 2015). Por consiguiente, resulta pertinente promover ambientes de aprendizajes vinculados sinérgicamente con procesos de resolución de problemas. En ese orden, las experiencias cotidianas de los estudiantes se pueden asociar a los conceptos químicos como elemento mediador del aprendizaje; evitando consigo dificultades conceptuales y epistemológicas (Adúriz-Bravo, 2017; Furió-Más y Furió, 2018). Así, las investigaciones en didáctica de la Química se han convertido en un insumo poderoso para develar nuevas formas de enseñanza sobre esta ciencia, tales como: enseñanza de la química mediante la contextualización, la indagación y la modelización (Caamaño, 2011, 2018; Izquierdo, 2017; Meroni et al., 2015) y el triplete químico como soporte teórico para la enseñanza disciplinar (Cutrera y Stipcich, 2016; Taber, 2019b; Talanquer, 2018b). El uso didáctico de este triplete químico genera en los estudiantes comprensión y reflexiones sobre las implicaciones sociales y ambientales que los rodean, desde un enfoque de aprendizaje en contexto (Ninou-Collado, 2017; Talanquer, 2018b).

En las últimas tres décadas ha crecido, por parte de la comunidad científica y académica, el interés en estudiar e implementar el triplete químico para orientar los procesos de enseñanza y aprendizaje contextualizado (Caamaño, 2018). Algunos autores muestran la importancia de interpretar correctamente el triplete químico, debido a que de esto depende la buena conducción de los procesos de comprensión y manejo crítico-reflexivo sobre los fenómenos estudiados (Caamaño, 2011, 2014, 2018; Cutrera y Stipcich, 2016; Freire et al., 2019; Furió-Más y Furió, 2018; Galagovsky et al., 2014; Galagovsky et al., 2003; Gilbert y Treagust, 2009; Mahaffy, 2011; Mahaffy et al., 2018; MenesesVillagrá et al., 2014; Ordenes et al., 2014; Taber, 2013, 2019b; Talanquer, 2011, 2018a). Desde el punto de vista de la didáctica de la Química, la propuesta del triángulo de Johnstone es una base teórica para la investigación sobre la enseñanza disciplinar (Cutrera y Stipcich, 2016). Otros autores como Caamaño (2014) muestran que es posible ver un triplete de la química, formado no solo por los conceptos y modelos mentales, así como sus representaciones, sino también por las entidades y procesos químicos reales. Taber (2013) re-describe el triplete original cuando se conceptualiza el nivel macro, para a partir de esto darle explicación con la conceptualización submicro, dejando fuera del triángulo el nivel simbólico para interconectar los otros niveles mediante el uso del vocabulario técnico. Con base en lo anterior, Caamaño (2014) propone que esta última idea se reestructure, aún más, ubicando en los vértices del triplete químico a las siguientes entidades: conceptos y modelos mentales, representación y realidad química. A su vez, Galagovsky et al. (2003) plantean que cuando los estudiantes se encuentran por primera vez con conceptos químicos sus sentidos ya no les permiten aprender mediante la percepción directa, debido a que son entes abstractos, mediados por interpretaciones simbólicas. Sobre la percepción de cualquier fenómeno, Furió-Más y Furió (2018) establecen que será filtrada ontológica y conceptualmente por el estudiante, basándose no solamente en su experiencia física, sino también en la cultura y el lenguaje cotidiano. Por todo ello, el presente estudio tiene como objetivo indagar las percepciones de maestros y estudiantes 
de Ciencias sobre nociones científicas, dominios u obstáculos acerca del uso del triplete químico y saltos entre los niveles representacionales durante los procesos de enseñanza y aprendizaje de la Química. De allí que la presente investigación girase en torno a los siguientes interrogantes principales: ¿cuál es la percepción de los maestros y estudiantes sobre el uso del triplete químico en la enseñanza y el aprendizaje de la Química? y ¿cuáles son los principales obstáculos y desafíos para la enseñanza y el aprendizaje de la Química contextualizada?

\section{Triplete químico en la enseñanza y el aprendizaje de la química contextualizada}

Durante los procesos de enseñanza y aprendizaje de la Química es común que los maestros y estudiantes empleen distintas formas para expresarse, lo que evidencia, caracteriza y diferencia el lenguaje utilizado por ellos al momento de representar fenómenos químicos (Galagovsky et al., 2003). Por lo general, los maestros conocen las representaciones en Química y pueden manejar el lenguaje científico, el cual plantea aspectos de los niveles submicro y simbólico que intentan explicar y trascender hasta el macro de los fenómenos estudiados (Sanchez, 2017). Sin embargo, las dificultades de los estudiantes para entender ciertas nociones químicas se derivan de su integración en el medio social y cultural. A su vez, las que se presentan en la comprensión del complejo mundo de la Química pueden deberse a incomprensiones en las interpretaciones macro o submicro de los fenómenos químicos estudiados (Furió-Más y Furió, 2018; Gabel, 1998). Ya que, los estudiantes tienden a disponer sobre "qué química aprender" y "para qué aprender química" el buen docente debe combinar los conocimientos disciplinarios, pedagógicos y didácticos para ayudar a pensar en química con el propósito de motivar, sorprender, despertar la curiosidad, generar interés y dar sentido por medio de la reflexión constante y consciente sobre los cononocimientos a enseñar (Talanquer, 2018a).

\section{Metodología}

En esta investigación se utilizó una metodología cualitativa cuyo objetivo fue describir e interpretar la realidad educativa desde adentro. Se basó, en especial, en la fenomenología (Barbera y Inciarte, 2012; Strauss y Corbin, 2002), la cual apunta al sujeto (maestros para este caso) como mediador del conocimiento construido a través de lo que se percibe de manera subjetiva y el estudiante (Talanquer, 2018a). Se utilizó la técnica de análisis de contenido cualitativo (ACC) (Krippendorff, 2009) y la teoría fundamentada (Friese, 2012; Glaser y Strauss, 1967; Strauss y Corbin, 2002), esto permitió formular a partir de ciertos datos inferencias reproducibles y válidas que fueran aplicables a un contexto (Friese, 2012).

\section{Participantes y contexto}

La indagación se llevó a cabo durante ocho semanas, por medio de grupos de discusión a estudiantes y entrevistas en profundidad a maestros de Ciencias en servicio. La muestra fue de tipo no probabilístico e intencionada, constituida por un total de tres grupos de nueve estudiantes y siete maestros de Ciencias de una escuela privada en Colombia. Para el análisis de los datos se usan los siguientes seudónimos para los estudiantes (E) participantes: E.1, E.2, E.3, E.4, E.5, E.6, E.7, E.8 y E.9; así mismo, los maestros (M) se identificaron como: M.1, M.2, M.3, M.4, M.5, M.6 y M.7.

\section{Análisis de datos}

El análisis de contenido utilizó ocho grabaciones de audio como fuentes de información: 86 minutos en entrevistas en profundidad a maestros de Ciencias y 186 minutos en grupos de discusión a estudiantes. En un primer nivel de análisis emergieron ciertos códigos que no se constituían aun como unidades de análisis, los cuales permitieron identificar presencias, frecuencias y recurrencias. Posteriormente, se categorizó la información y se 
produjo una clasificación semántica para identificar categorías y subcategorías analíticas emergentes. Estos procesos se realizaron utilizando el software Atlas.ti, versión 8.4.4. para la organización, análisis e interpretación de los datos (Verdú y Chica, 2015, p. 127). La información obtenida se contrastó con un análisis documental para conocer la relación del triplete químico en los procesos de enseñanza y aprendizaje de la Química contextualizada. Para el tratamiento de los datos se describieron pasos sucesivos y sistemáticos, estableciendo condiciones que aseguraran la consistencia y coherencia de los resultados obtenidos (Strauss y Corbin, 2002; Verdú y Chica, 2015). La figura 1 describe las etapas que se consideraron en el plan de análisis de los datos, por medio del cual se organizó la información en las siguientes fases: preanálisis, codificación y categorización.

\section{Plan de análisis de datos}

En la primera etapa de preanálisis se preparó el contenido de la grabación a analizar, obtenido de los grupos de discusión y entrevistas en profundidad, esto dio origen a las unidades hermenéuticas en las que se siguieron reglas analíticas de contenido y modelos paso a paso sin utilizar cuantificación (Friese, 2012; Strauss y Corbin, 2002).

\section{Reducción y análisis de los datos}

Este momento del análisis consistió en encontrar relaciones entre los datos en distintos niveles de codificación. Para ello se efectuaron operaciones analíticas de codificación y categorización de los mismos para descubrir y desarrollar los conceptos contenidos en los audios, exponiendo los pensamientos, ideas y significados (Strauss y Corbin, 2002).

En esta etapa se agruparon códigos similares (familias temáticas), descartándose algunos conceptos que no tenía relación con los objetivos de la investigación; aunque en esta fase de análisis no suponían ser categorías explicativas.

\section{Fase 1. Codificación de las unidades de análisis. Codificación abierta}

En esta etapa se procedió agrupar y separar el contenido específico, de modo que fuera pertinente para el objeto de estudio de la investigación. Acá se necesita que los insumos guardados tengan

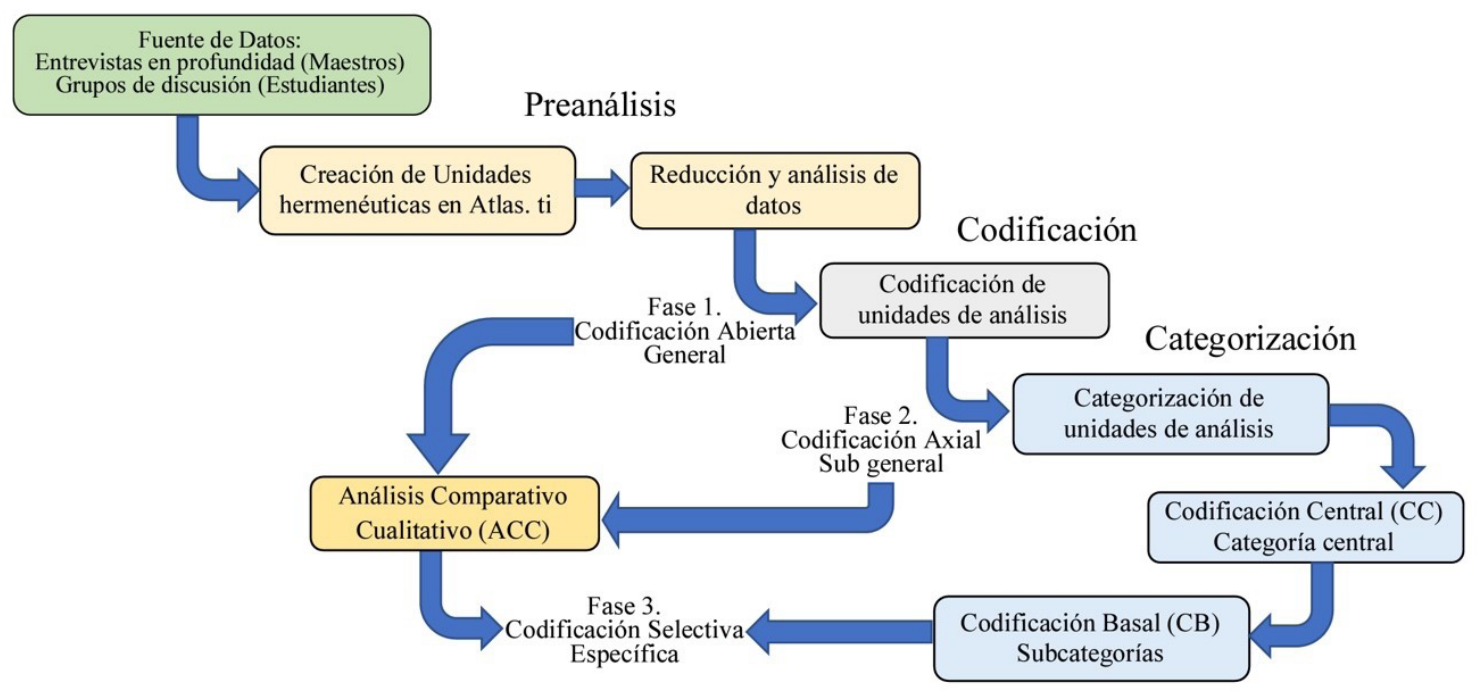

Figura 1. Plan de análisis de datos.

Fuente: elaboración propia según Strauss y Corbin (2002). 
relación suficiente como para ser considerados similares; por el contrario, se pueden considerar que aquellos que forman parte de otros conjuntos de datos conformando otros grupos.

El propósito de las acciones anteriores fue crear segmentos o citas en la unidad hermenéutica, lo que permitió descubrir tanto en los grupos de discusión con estudiantes como las entrevistas con maestros de Química en servicio patrones conversacionales en sus respuestas.

\section{Fase 2. Categorización de las unidades de análisis. Codificación Axial}

Esta categorización corresponde a un segundo nivel de análisis. Se clasificaron los códigos de citas dentro de todo el conjunto realizado, a partir de ciertos criterios previamente definidos por medio de razonamiento inductivo. Las categorías en la unidad hermenéutica fueron los segmentos que reúnen un conjunto de códigos de citas emergentes bajo un mismo criterio de selección o semejanza según sea el objeto de análisis.

\section{Codificación central (CC). Categoría Central}

El proceso de codificación central o categorías centrales tiene en cuenta los datos preliminares en la fase 1 del plan de análisis de los datos (figura 1). En esta etapa se incluye la categorización previa, sistematización y el análisis de todos los datos generados en el proceso de investigación (Strauss y Corbin, 2002).

\section{Categorización basal (CB). Subcategorías}

Para la selección de las subcategorías se realizó un análisis de segundo orden a partir de las codificaciones anteriores (emergentes) y se constituyó como el centro de esta investigación. Las categorías de análisis permitieron dar información sobre el planteamiento del problema, además de evidenciar otros problemas no previstos en la estructura de la investigación.

\section{Fase 3. Codificación Selectiva}

Posterior a la codificación axial, en la cual se relacionan las categorías con sus respectivas subcategorías y códigos siguiendo también sus características y propiedades afines, se llegó al proceso de codificación selectiva (Verdú y Chica, 2015).

\section{Resultados y discusiones}

La discusión de resultados se desarrolló por categorías y subcategorías, las cuales son mostradas en la tabla 1, mediante comparaciones con otras fuentes de análisis global o fundamentos teóricos.

Posteriormente, se realizaron las redes semánticas según las relaciones que se pudieron evidenciar en los datos y en la afinidad entre los mismos, a través del proceso de análisis de contenido. En esta se muestra el grado de importancia de dos categorías centrales de análisis para maestros y tres para estudiantes (figura 2).

\section{Categoría 1. Representaciones en Química (maestros)}

En el análisis de las entrevistas en profundidad a maestros de Ciencias, y en especial de Química, algunos manifestaron desconocimiento de las formas de enseñanza de la Química mediante el triplete químico; lo que genera obstáculos en los procesos de enseñanza y aprendizaje de esta ciencia. En la figura 3 se muestra la red semántica para la categoría representaciones en química.

$\mathrm{Al}$ respecto, algunos maestros manifiestan:

He leído documentación, pero ubicarlos específicamente no; tengo insumos bibliográficos, pero no los categorizo como deberían hacerse normalmente. (M.1)

Otros expresan:

¿Representaciones en química de acuerdo a lo que se les explica o se les enseña a los estudiantes en el aula? (M.2) 
Tabla 1. Resultados de la codificación axial, derivada del procesamiento de la información

\begin{tabular}{|c|c|c|c|c|}
\hline \multirow[t]{9}{*}{ Categoría } & Subcategoría & Código & G.D. & E.M. \\
\hline & Macro & Percepción & $\mathrm{x}$ & $\mathrm{x}$ \\
\hline & Macro & Subjetivo & $x$ & \\
\hline & Macro & Experimental & $x$ & $\mathrm{x}$ \\
\hline & Macro & Variabilidad orgánica & $x$ & \\
\hline & Macro & Propiedades organolépticas & $x$ & $x$ \\
\hline & Submicro & Átomos y moléculas & $x$ & $x$ \\
\hline & Submicro & Cargas eléctricas & $x$ & \\
\hline & Submicro & Hibridación & $x$ & $x$ \\
\hline \multirow[t]{15}{*}{ Nociones químicas } & Submicro & Imaginación & $x$ & $\mathrm{x}$ \\
\hline & Simbólico & Lenguaje gráfico & $x$ & $\mathrm{x}$ \\
\hline & Simbólico & Lenguaje visual & $\mathrm{x}$ & $\mathrm{x}$ \\
\hline & Simbólico & Lenguaje verbal & $x$ & $x$ \\
\hline & Simbólico & Cantidades & $x$ & $x$ \\
\hline & Simbólico & Estadística & $x$ & $x$ \\
\hline & Simbólico & Análisis de datos & $x$ & $x$ \\
\hline & Simbólico & Ecuaciones & $x$ & $x$ \\
\hline & Simbólico & Enlaces & $x$ & \\
\hline & Simbólico & Composición química* & $x$ & $x$ \\
\hline & Macro-simbólico & Saltos entre niveles* & $x$ & \\
\hline & Representaciones en química & Conocimientos químicos & $x$ & $x$ \\
\hline & Representaciones en química & Nomenclatura química & $x$ & \\
\hline & Representaciones en química & Síntesis orgánica & $x$ & \\
\hline & Representaciones en química & Relación con otras ciencias & $x$ & $x$ \\
\hline \multirow{9}{*}{$\begin{array}{l}\text { Qué Química } \\
\text { aprender }\end{array}$} & Representaciones en química & Valorar limitaciones & $x$ & $x$ \\
\hline & Influencia antropogénica & Problemas ambientales & $x$ & \\
\hline & Influencia antropogénica & Solución a problemas contextuales & $x$ & $x$ \\
\hline & Influencia antropogénica & Conciencia ambiental & $x$ & $x$ \\
\hline & Influencia antropogénica & Proyectos & $x$ & \\
\hline & Influencia antropogénica & Potenciar aprendizajes & $x$ & $x$ \\
\hline & Influencia antropogénica & Vincular a los estudiantes* & $x$ & $x$ \\
\hline & Aplicabilidad química & Aprendizajes aplicables & $x$ & $x$ \\
\hline & Aplicabilidad química & Aplicabilidad industrial & $x$ & \\
\hline \multirow{7}{*}{$\begin{array}{l}\text { Para qué aprender } \\
\text { Química }\end{array}$} & Aplicabilidad química & Actitudes negativas & $x$ & $x$ \\
\hline & Aplicabilidad química & Habilidades de aprendizajes & $x$ & $x$ \\
\hline & Comprensión de fenómenos & Solución a problemas contextuales & $x$ & $x$ \\
\hline & Comprensión de fenómenos & Conciencia ambiental & $x$ & \\
\hline & Comprensión de fenómenos & Concepciones alternativas & $x$ & $x$ \\
\hline & Comprensión de fenómenos & Caracterizar al estudiante* & $x$ & $x$ \\
\hline & Comprensión de fenómenos & Cambiar enseñanza tradicional* & $\mathrm{x}$ & $\mathrm{x}$ \\
\hline
\end{tabular}

Nota: esta tabla muestra las categorías, subcategorías y códigos obtenidos del proceso de análisis de los datos provistos de los grupos de discusión a estudiantes (G.D.) y entrevistas en profundidad a maestros de Ciencias (E.M). Además, se presenta la relación entre los códigos emergentes.

*Estos códigos cobran importancia para esta investigación cuando están relacionados con otros códigos.

Fuente: elaboración propia de los autores. 


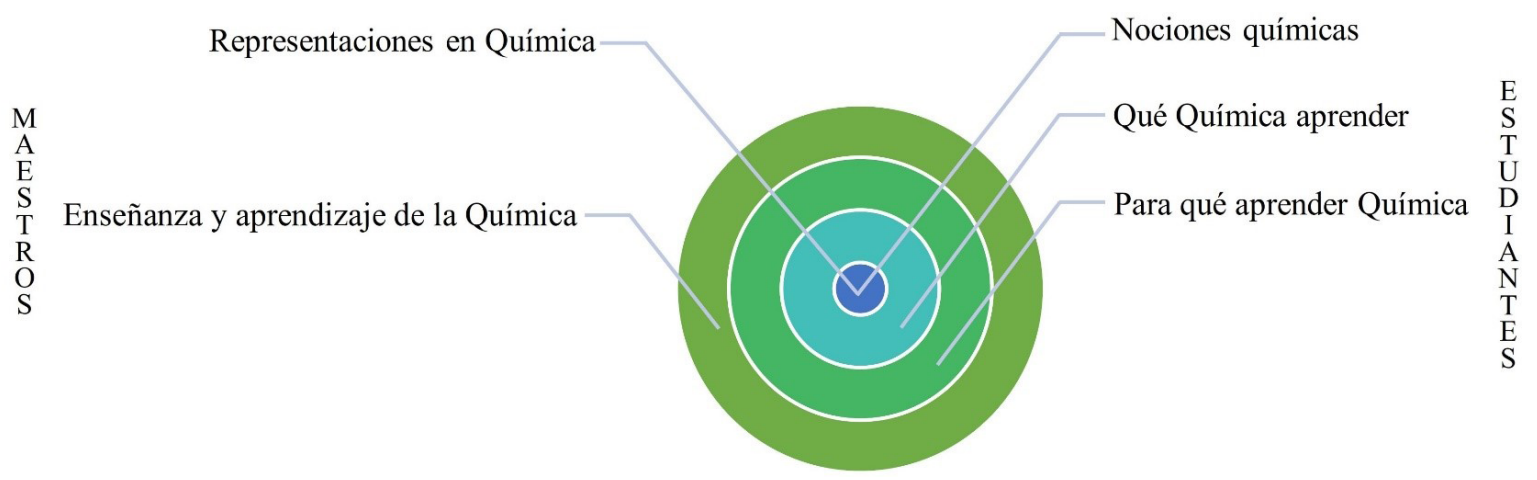

Figura 2. Categorías centrales obtenidas del plan de análisis de los datos de las entrevistas en profundidad a maestros y grupos de discusión a estudiantes.

Fuente: elaboración propia de los autores.

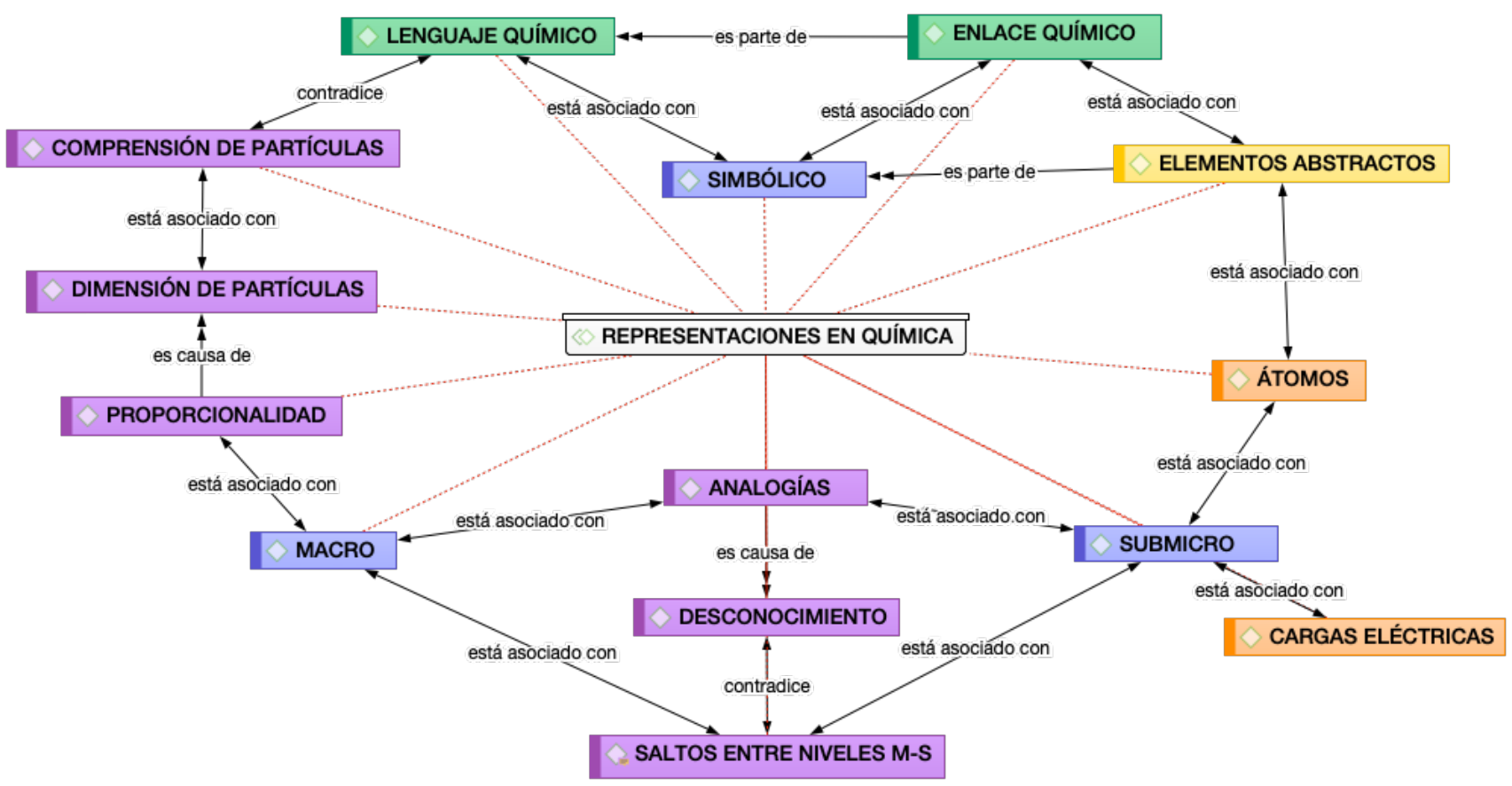

Figura 3. Red semántica sobre representaciones en Química. Perspectivas de maestros en ejercicio.

Fuente: elaboración propia de los autores.

Lo que entiendo de representaciones en química es lo que pueden ver los estudiantes y lo que no pueden ver. (M.3)

Sin embargo, pese a lo evidente en las declaraciones de los maestros, es notable que, como profesionales, ejercen una función de responsabilidad social sobre los estudiantes. A su vez, la mayoría de los maestros de Ciencias adquieren conocimientos básicos de química en cursos con contenidos meramente disciplinarios, por lo que su preparación pedagógica y didáctica es resultado de su participación en cursos de educación con carácter general (Talanquer, 2018a). 
Por otra parte, los maestros en las entrevistas suscitan el conocimiento químico, interpretando en algunos casos de forma errónea los niveles representacionales y, en otros, la repercusión que tiene estos sobre los procesos de aprendizaje en los estudiantes. Sin embargo, no son conscientes que sobre ellos recae la responsabilidad del conocimiento químico, aunque eso no signifique que fueran el centro del proceso de enseñanza y aprendizaje. Después de lo anterior expuesto, ¿hasta que punto los maestros son conscientes que sus procesos de enseñanza no son eficientes para la educación química actual? Algunos maestros entrevistados expresan:

Yo me imagino que ellos [estudiantes] hacen una visión de las cosas que se les explica en clase [...] no podemos ver un átomo, pero podemos representarlo o un espectro de lo que son los movimientos y hay instrumentos electrónicos que nos pueden ayudar a eso. (M.6)

Sobre este punto, los maestros "suponen" que los estudiantes modelan en su mente las representaciones macro y simbólicas. No obstante, debido a los errores en la interpretación de los niveles del triplete químico el maestro puede transferir a los estudiantes esos errores conceptuales y epistemológicos de los procesos químicos estudiados.

Por otro lado, los maestros reconocen que en el nivel submicro hay "elementos abstractos" y manifiestan la importancia de generar un conocimiento que involucre la comprensión de los mismos en los estudiantes. Sobre esto el M.4 expresó:

[...] en química algunos elementos son muy abstractos, sin embargo, es necesario generar en nuestros estudiantes la importancia de lo que es el sentido de tener claridad sobre las partículas involucradas en un fenómeno.

Asimismo, para los estudiantes el mundo submicro (átomos, moléculas, cargas eléctricas) es el mismo mundo macro que perciben con los sentidos, pero en "diminuto" (Furió-Más y Furió, 2018). Por tanto, no comprenden que existen distintos niveles representacionales de la materia en íntima relación: el nivel macro de las sustancias con sus propiedades y cambios y, por otra parte, el nivel submicro de aquellas mismas sustancias que la Química modela a base de átomos (Furió-Más y Furió, 2018).

\section{Categoría 2. Enseñanza y aprendizaje de la Química (maestros)}

En esta categoría (figura 4) se evidencia que los maestros son conscientes de las dificultades que los estudiantes presentan frente al conocimiento químico. Igualmente, manifiestan la necesidad de reestructurar los procesos de enseñanza, desde el punto de vista contextualizado de la Química, para mejorar las actitudes negativas de los estudiantes frente a esta ciencia. Un maestro expresa:

La gran mayoría de nuestros estudiantes se quedan en una representación visual, que no llega a su memoria a largo plazo. (M.7)

Además, los entrevistados señalan que es necesario emplear metodologías de enseñanza que generen conflicto cognitivo desde los saberes previos de los estudiantes. Sobre lo anterior el M.6. expresa:

Los saberes previos de los estudiantes, son el punto de partida para la enseñanza de las ciencias, pues es lo que da sentido, y favorece que adquieran aprendizajes significativos.

En efecto, se infiere que los maestros involucran la memoria perceptiva y los saberes previos de los estudiantes para condicionar los conceptos químicos que aprenden (Galagovsky et al., 2003). Sin embargo, los estudiantes que van a iniciar un curso de Química por lo general vienen con saberes previos de esta ciencia; usualmente saberes populares generados por la interacción de la sociedad con los medios de comunicación que promueven una reputación falsa de esta disciplina científica (Ordaz y Mostue, 2018). 


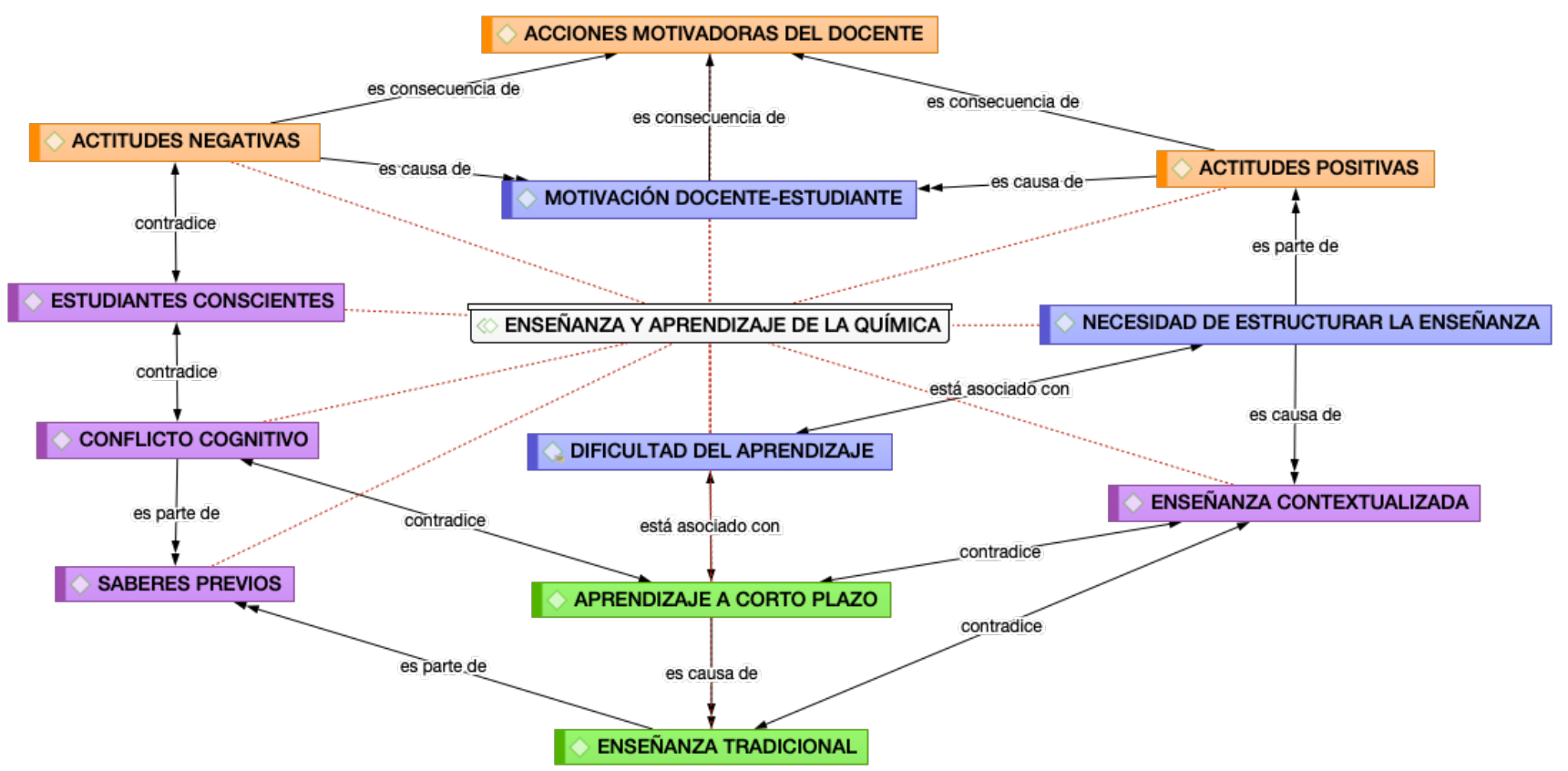

Figura 4. Red semántica sobre enseñanza y aprendizaje de la Química, perspectivas de maestros en ejercicio.

Fuente: elaboración propia de los autores.

No obstante, la mayoría de los maestros entrevistados expresan que desarrollan prácticas de enseñanzas tradicionales, basadas en la transmisión del conocimiento, contenidos procedimentales y metodologías que se ponen en funcionamiento con base en libros de texto de Química y lineamientos curriculares dispuestos por el Ministerio de Educación Nacional de Colombia (MEN). En este aspecto el M.5 relata:

La Química se enseña con base en lineamientos curriculares impuestos por el MEN, y generalmente, atienden a una prueba nacional y no para ser aplicada al contexto del estudiante.

Lo anterior implica que la educación en Química debe propiciar acciones encaminadas a que los maestros se capaciten en procesos y metodologías de enseñanza situadas desde el contexto de los estudiantes que además permitan realizar cambios profundos en el currículo escolar (Caamaño, 2018). También, los maestros conciben la enseñanza como un proceso de "recepción", "adquisición" o "acumulación" de conocimientos en donde le apuestan por contenidos predispuestos en un currículo, muchas veces presentados de forma descontextualizada, que deben ser igualmente asimilados por todos los estudiantes tal cual son dados y desconociendo las individualidades de los procesos formativos (Ordaz y Mostue, 2018).Sobre esto, el M.8 expresa:

Los estudiantes se les enseña a partir de los textos, generalmente en el aula de clase por medio de clases magistrales.

Se evidencia, entonces, el énfasis que se hace en la enseñanza basada en contenidos y que no se tienen en cuenta los conceptos de la Química desde un enfoque por competencias (Izquierdo, 2017).

\section{Categoría 1. Nociones químicas (estudiantes)}

En la figura 5 se muestra la categoría central nociones químicas en los estudiantes. Se observa que los estudiantes emplean los niveles representacionales 
y clasifican cada uno según sus nociones. Para el caso del nivel macro, suscitan que son las formas de percibir y observar los fenómenos. Sin embargo, expresan que es un nivel en el cual se puede incurrir en subjetividades, porque en la mayoría de los casos no se tiene una herramienta más allá de los sentidos para interpretarlos.

Al respecto, algunos estudiantes utilizan el nivel macro como punto de partida para comprender el fenómeno, el E.4 expresa:

[...] hay que tener en cuenta que todo lo que conforma las partículas elementales y los símbolos químicos, van a tener consecuencia en lo que percibimos, entonces ¿por qué se utiliza primero lo que percibimos? Porque sus efectos se logran ver a simple vista, entonces nosotros no podemos ver las reacciones que se dan, pero si podemos ver las consecuencias físicas y fenotípicas que se representan.

De esta manera, se deja al descubierto que los estudiantes son capaces de interpretar el fenómeno en términos de la formación de nuevas sustancias. Cabe resaltar que los estudiantes hacen uso de representaciones macro, referidas en este caso a aquello que perciben con sus sentidos debido a que la mayoría de los conceptos que les resultan familiares tienen algo de tangible, algo que puede ser reconocido por sus sentidos. Por tanto, el aprendizaje de este tipo de conceptos les resulta más sencillo que el de conceptos abstractos (Galagovsky et al., 2003).

En ese orden, el nivel macro es el primer recurso de los estudiantes por ser el más accesible, convirtiéndose en prioritario. Sin embargo, los conceptos o representaciones mentales se construyen a partir de las imágenes sensibles cuando se trata de objetos, sustancias o procesos macro; pero también a partir de las representaciones simbólicas, icónicas y formales cuando se trata de interacciones, procesos o propiedades submicro (Caamaño, 2014, p. 13). En efecto, los estudiantes emplean el nivel submicro a partir de modelos mentales que construyen producto de su imaginario; pues, asocian estas representaciones con nociones químicas provistas de sus procesos de aprendizaje en el aula o contenidos de textos escolares. No obstante, tienden a confundir las representaciones submicro con simbólicas y macro cuando describen la formación de cargas eléctricas con la representación

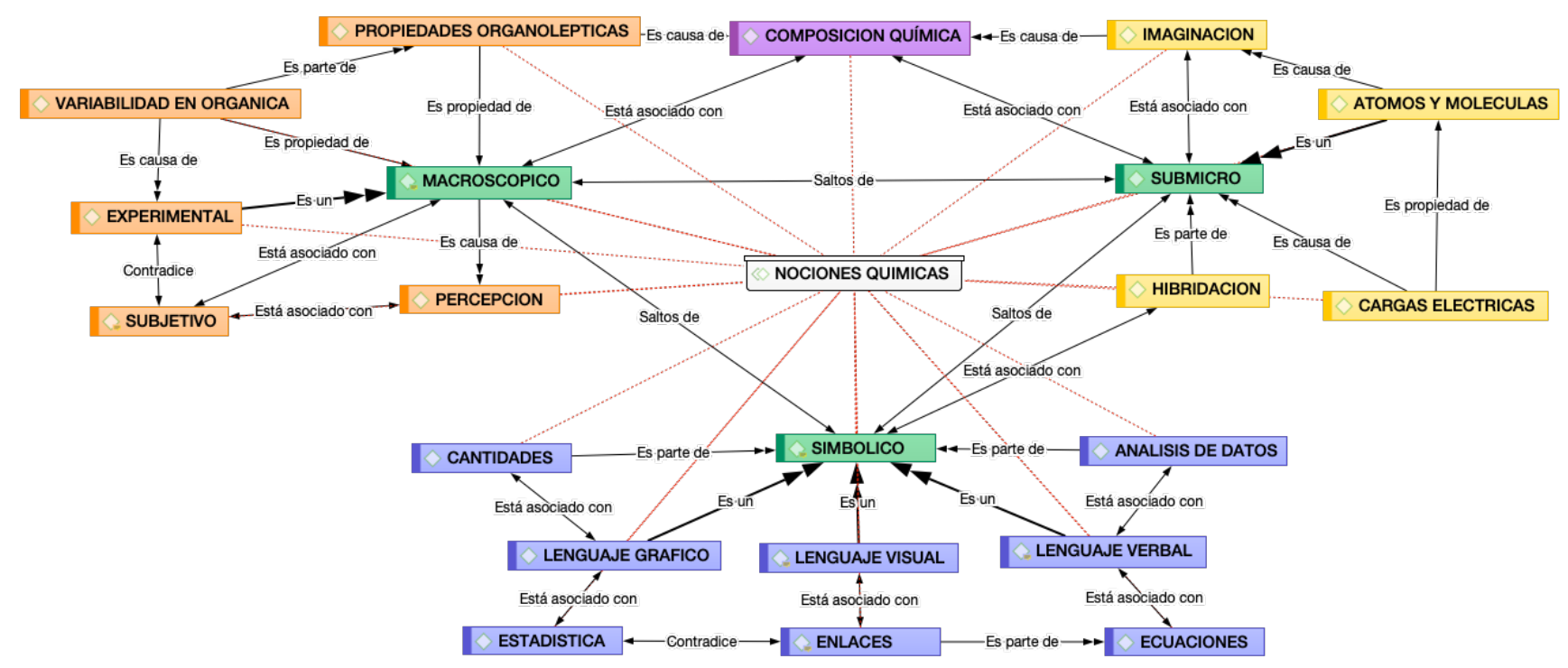

Figura 5. Red semántica de nociones químicas de los estudiantes de la muestra.

Fuente: elaboración propia de los autores. 
de los enlaces químicos (guion entre elementos) y sus propiedades observables. Adicionalmente, los estudiantes durante su proceso de aprendizaje de conceptos químicos atraviesan por un nivel representacional intermedio, erróneo, entre el macro y el submicro (Galagovsky et al., 2003, p. 111). Por tanto, es importante que el maestro emplee una movilidad mental entre los niveles macro, submicro y simbólico; asimismo, que pueda proveer una enseñanza focalizada y más genuina en los estudiantes, permitiéndoles comprender la naturaleza de la Química de forma apropiada.

Por último, el nivel simbólico es descrito por los estudiantes como el "manejo" del fenómeno desde las ecuaciones y cantidades numéricas que participan en una reacción química. Esto indica que los estudiantes intentan describir un fenómeno por medio de expresiones simbólicas al realizar representaciones de fórmulas y ecuaciones químicas. Además, consideran estas representaciones como indicativos de una reacción o cambio químico que pudieran estar presentándose en el fenómeno. Algunos estudiantes declaran:

Con las ecuaciones, es en donde se puede demostrar, por así decirlo, la veracidad o no de cierta teoría representada. (E.9)

Aunque se pueda observar un fenómeno, puedo explicarlo mejor con un método gráfico, en donde puedo analizar detalladamente el proceso o suceso que se está desarrollando. (E.2)

También, expresan que el lenguaje es algo útil para estos procesos. El E.3 expresa:

¿Qué nos permiten las fórmulas y las ecuaciones? representar en un lenguaje que sea comprensible y que facilite el entendimiento para los demás...

En este último aspecto el nivel simbólico se interrelaciona con los otros niveles representacionales mediante el uso del vocabulario técnico y las representaciones formales de la Química (Taber, 2013, p. 156). Sin embargo, la complejidad del lenguaje químico es uno de los obstáculos para el aprendizaje de conceptos propios de esta ciencia por parte de los estudiantes (Galagovsky et al., 2014, p. 785).

\section{Categoría 2. Qué Química aprender (estudiantes)}

De acuerdo con los relatos procesados para la red semántica, la categoría central representaciones en Química (figura 6) guarda una fuerte relación con el aprendizaje de la Química, puesto que los estudiantes se expresan críticamente al referirse al sistema educativo. El E.1 expresa en este punto:

Las personas que están en un mandato mayor no nos limitan, es decir, no nos pueden retener nuestras ideas.

A su vez, perciben el triplete químico como una forma de desglosar la química para su mejor comprensión. Lo que permitiría conformar subconjuntos de la Ciencia, hecho que se puede sustentar cuando expresaban:

Yo opino que sencillamente son una forma de desglosar la química para entenderla en una forma más puntual y manejable para cada estudiante. (E.6)

Por otro lado, los estudiantes asocian a la subcategoría influencia antropogénica con la participación del ser humano como agente principal en los cambios de su entorno. Esto confirma lo que Tamayo et al. (2015, p. 112) establecen al identificar estas reflexiones críticas como uno de los pilares fundamentales de la educación, pues aportan a la formación del pensamiento crítico general y dominio específico en los estudiantes. Sobre lo anterior el estudiante E.9 expresa:

El conocimiento científico del ser humano, no solo lo usa para su beneficio, está también afectando las cosas que nos rodean de forma irreversible, en algunos casos. 


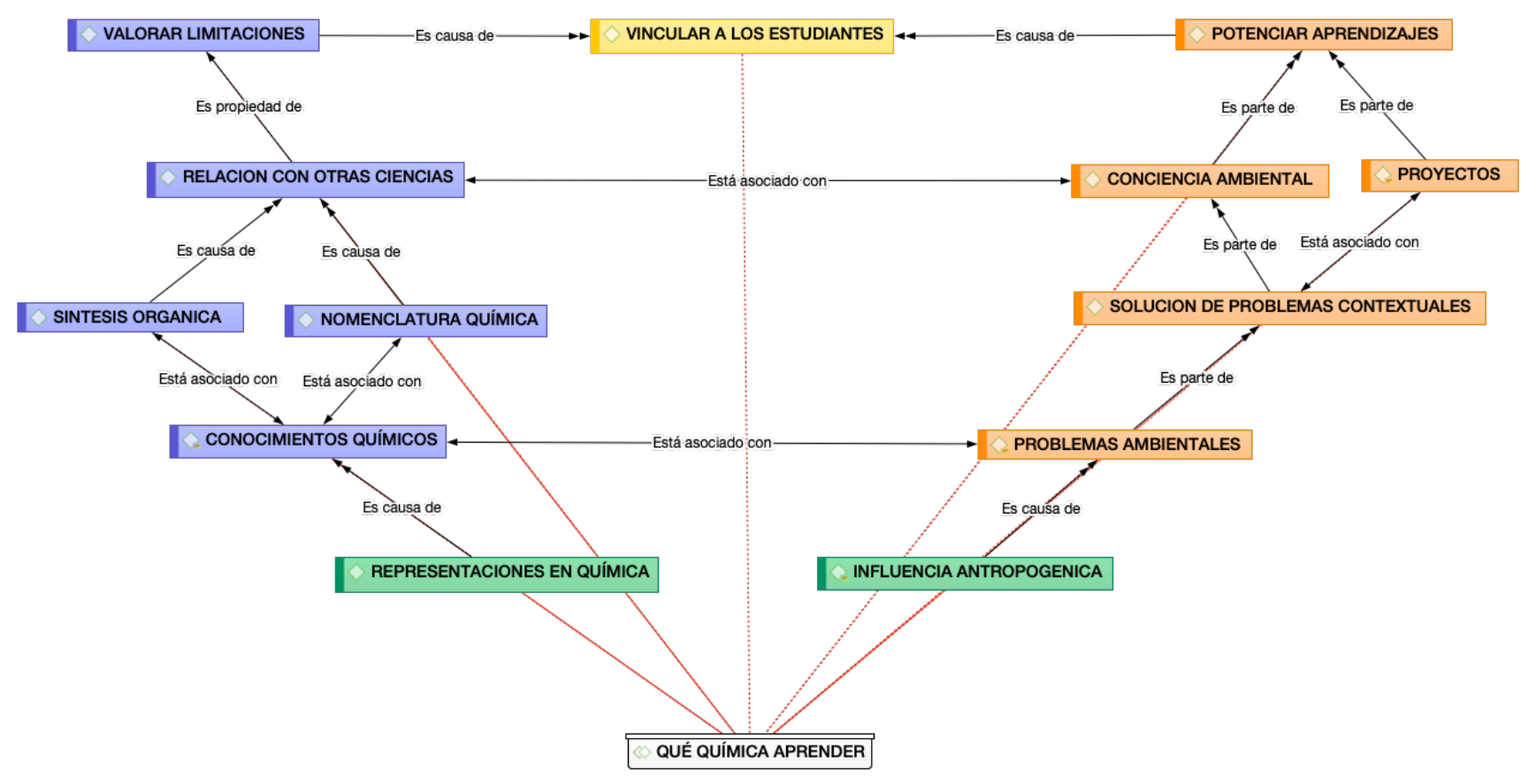

Figura 6. Red semántica de la categoría central qué Química aprender.

Fuente: elaboración propia de los autores.

Por tanto, el contexto sociocultural tiene influencia circunstancial en las representaciones que forman los estudiantes de esta ciencia y el contexto donde interactúan (Perera, 2003).

\section{Categoría 3. Para qué aprender Química (Estudiantes)}

De acuerdo con los resultados de la codificación, en la categoría emergente de la figura 7 se hizo una aproximación teórica con el fin de adentrarse en la discusión. Es importante resaltar que la Química siempre ha estado presente en la existencia del hombre para satisfacer sus necesidades y mejorar su calidad de vida (Montes, 2015, p. 72). La subcategoría aplicabilidad química muestra que los estudiantes sugieren vencer los "estigmas" como atributo profundamente desacreditador que la Química tiene como ciencia, en cuanto a su aprendizaje y a su aplicabilidad. Sobre este punto la E.4 expresa:
La idea es romper con esa barrera, y que se entienda y se comprenda la química como lo que es, como algo cotidiano y necesario en la sociedad.

Por tanto, las representaciones sociales de los estudiantes hacia la Química se componen desde lo cognitivo como un conjunto de teorías que permiten al estudiante solucionar problemas de su cotidianidad, al tiempo que las consideran útiles y aplicables (Meneses-Villagrá et al., 2014; Perera, 2003).

Por otro lado, los estudiantes expresan su interés por la comprensión de fenómenos; un punto relevante para este estudio pues atañe directamente al maestro de Ciencias debido a que son quienes ponen en marcha metodologías de enseñanza de la Química. Aun así, por lo general la Química se enseña en las instituciones de educación media por medio de contenidos que se encuentran alejados de los intereses de los estudiantes y de los problemas contextuales (Caamaño, 2006). Sobre 


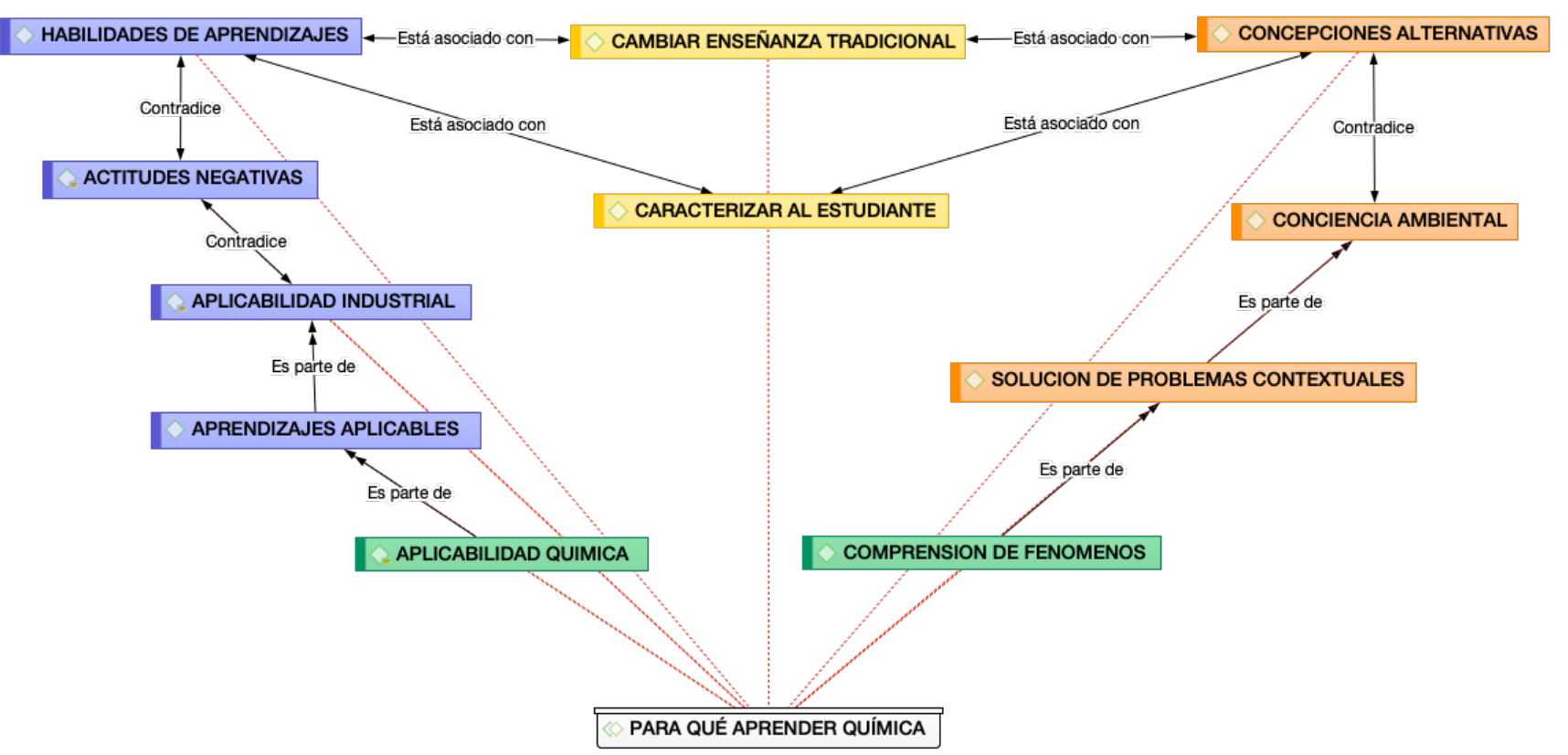

Figura 7. Red semántica de la categoría central para qué aprender Química.

Fuente: elaboración propia de los autores.

la base de las consideraciones anteriores, un estudiante expresa:

Los fenómenos socioambientales se perciben con los sentidos, entonces al percibirse se puede inferir o tratar de buscar el por qué de esa problemática. (E.4)

Por consiguiente, es evidente la importancia que el estudiante le confiere a la comprensión de fenómenos de su entorno, utilizando en algunos casos el triplete químico. Así, desde un enfoque por competencias de la Química pueden mediarse aprendizajes profundos en los estudiantes, como una alternativa metodológica de enseñanza, lo que le permitiría que construyan el camino apropiado para desenvolverse adecuadamente en su contexto.

\section{Conclusiones}

El estudio evidenció que los maestros seleccionados en esta investigación no son conscientes de las interrelaciones o saltos en los niveles del triplete químico. Algunos los desconocen y otros crean interpretaciones erróneas en los procesos de enseñanza. Esto genera que los estudiantes no comprendan la Química, ni tampoco el fenómeno que están percibiendo, lo que provoca actitudes negativas hacia esta ciencia. Por tanto, se hace necesario orientar la enseñanza de la Química no solo hacia su contextualización, sino también que los maestros usen, interpreten correctamente y sean conscientes del triplete químico como un contenido conceptual eficiente y poderoso en educación química.

Con respecto a las percepciones de los estudiantes, se observa que el uso de los niveles macro y simbólico son característicos para comprender y explicar fenómenos del contexto. Dichas representaciones arrojan luces sobre aspectos relacionados con aquellas características que son observables. Además, los estudiantes son capaces de interpretar el fenómeno en términos de la formación de nuevas sustancias, utilizando un lenguaje impreciso e indiferenciado para expresar sus nociones frente a los conceptos químicos. 
Los principales obstáculos encontrados confieren al presente trabajo un estatus de estudio inicial. El desafío será complementar con investigaciones posteriores en las cuales se pretenda trabajar sobre propuestas didácticas que involucren la apropiación, usos y correcta interpretación del triplete químico (macro, submicro y simbólico), mediante el estudio de fenómenos contextualizados específicos. Esto implicaría enlazar el aprendizaje para la comprensión de cualquier objeto de estudio con enfoques constructivistas, en donde se pueda concebir el aprendizaje como una amalgama de información, por medio del cual tienen gran relevancia las interacciones y el diálogo del maestro y los estudiantes. Por lo anterior, los procesos de enseñanza y aprendizaje de la Química instan a generar profundas transformaciones en aras de contribuir a las necesidades educativas que, desde la educación temprana hasta la educación superior, requieren para abordar enfoques no tradicionalistas. Lo anterior conlleva que el maestro abandone el enfoque de transmisión de conocimientos, la enseñanza basada en contenidos y tome consciencia sobre la contextualización de fenómenos a partir del triplete químico con el objetivo de que pueda converger sobre su labor y el sentir de los estudiantes.

Desde el punto de vista práctico, la labor del maestro debe trascender el aula de clases e ir encaminada a crear las condiciones apropiadas para que los estudiantes construyan y reflexionen sobre los aprendizajes adquiridos, desde posturas críticas y transformadoras para su contexto.

\section{Referencias}

Adúriz-Bravo, A. (2017). Desafíos de la enseñanza de la epistemología al profesorado de ciencias. En Obstáculos epistemológicos en la enseñanza y el aprendizaje de la Filosofía y de la Ciencia (pp. 51-68). Universidad Nacional Autónoma de México.

Barbera, N., Inciarte, A. (2012). Fenomenología y hermenéutica: dos perspectivas para estudiar las ciencias sociales y humanas. Multiciencias, 12(2), 199-205.

Caamaño, A. (2006). Repensar el currículum de química en el bachillerato. Educación Química, 17(2).

Caamaño, A. (2011). Enseñar Química mediante la contextualización, la indagación y la modelización. Alambique, Didáctica de las Ciencias Experimentales, 69, 21-34.

Caamaño, A. (2014). La estructura conceptual de la Química: realidad, conceptos y representaciones simbólicas. Alambique: Didáctica de las Ciencias Experimentales, 78, 7-20.

Caamaño, A. (2018). Enseñar Química en contexto: un recorrido por los proyectos de Química en contexto desde la década de los 80 hasta la actualidad. Educación Química, 29(1), 21. https:// doi.org/10.22201/fq.18708404e.2018.1.63686

Cutrera, G., Stipcich, S. (2016). El triplete químico. Estado de situación de una idea central en la enseñanza de la Química. Revista Electrónica Sobre Cuerpos Académicos y Grupos de Investigación en Iberoamérica, 3(6), 1-24.

Freire, M., Talanquer, V., Amaral, E. (2019). Conceptual profile of chemistry: a framework for enriching thinking and action in chemistry education. International Journal of Science Education, 41(5), 674-692. https://doi.org/10.108 $\underline{0 / 09500693.2019 .1578001}$

Friese, S. (2012). Qualitative Data Analysis with Atlas.ti. En Qualitative Research (pp. 382-384). https://doi.org/10.1177/1468794113475420

Furió-Más, C. J., Furió, C. (2018). Dificultades conceptuales y epistemológicas en el aprendizaje de los procesos químicos. Educación Química, 11(3), 300. https://doi.org/10.22201/ fq.18708404e.2000.3.66442

Gabel, D. (1998). The Complexity of Chemistry and Implications for Teaching. En International Handbook of Science Education (pp. 233-248). Springer Netherlands. https://doi. org/10.1007/978-94-011-4940-2 15

Galagovsky, L., Bekerman, D., Giacomo, M. A., Di Alí, S. (2014). Algunas reflexiones 
sobre la distancia entre "hablar química" y "comprender química". Ciência y Educação (Bauru), 20(4), 785-799. https://doi. org/10.1590/1516-73132014000400002

Galagovsky, L. R., Rodríguez, M. A., Stamati, N., Morales, L. F. (2003). Representaciones mentales, lenguajes y códigos en la enseñanza de ciencias naturales. Un ejemplo para el aprendizaje de concepto de "reacción química" a partir del concepto de "mezcla". Enseñanza de las Ciencias: Revista de Investigación y Experiencias Didácticas, 21(1), 107-121.

Gilbert, J. K., Treagust, D. F. (2009). Towards a Coherent Model for Macro, Submicro and Symbolic Representations in Chemical Education (pp. 333-350). https://doi. org/10.1007/978-1-4020-8872-8 15

Glaser, B., Strauss, G. (1967). The Discovery of Grounded Theory: Strategies for Qualitative Research. American Journal of Sociology, 73, 773-774. https://doi.org/10.1086/224572

Izquierdo, M. (2017). Atando cabos entre contexto, competencias y modelización. ¿Es posible enseñar ciencias a todas las personas? Modelling in Science Education and Learning, 10(1), 309. https://doi.org/10.4995/msel.2017.6637

Johnstone, A. (1982). Macro-and micro-chemistry. En School Science Review, 64(227), 377-379.

Johnstone, A. H. (1991). Why is science difficult to learn? Things are seldom what they seem. Journal of Computer Assisted Learning, 7(2), 75-83. https://doi.org/10.1111/j.1365-2729.1991. tb00230.x

Johnstone, A. H. (1993). The development of chemistry teaching: A changing response to changing demand. Journal of Chemical Education, 70(9), 701. https://doi.org/10.1021/ed070p701

Johnstone, A. H. (2000). Teaching of Chemistry - Logical or Psychological? Chemistry Education Research and Practice, 1(1), 9-15. https://doi.org/10.1039/a9rp90001b

Krippendorff, K. (2009). The content analysis reader. Sage Publications.
Mahaffy, P. (2011). The Human Element: Chemistry Education's Contribution to Our Global Future. En The Chemical Element: Chemistry's Contribution to Our Global Future: First Edition (pp. 131-157). https://doi.org/10.1002/9783527635641.ch4

Mahaffy, P. G., Krief, A., Hopf, H., Mehta, G., Matlin, S. A. (2018). Reorienting chemistry education through systems thinking. Nature Reviews Chemistry, 2(4), 0126. https://doi.org/10.1038/s41570-018-0126

Meneses-Villagrá, J. Á., Lacolla, L., Valeiras, N. (2014). Reacciones químicas y representaciones sociales de los estudiantes. Enseñanza de las Ciencias, 32(3). https://doi.org/10.5565/rev/ensciencias.1010

Meroni, G., Copello, M. I., Paredes, J. (2015). Enseñar Química en contexto. Una dimensión de la innovación didáctica en educación secundaria. Educacion Quimica, 26(4), 275-280. https://doi.org/10.1016/j.eq.2015.07.002

Montes-Valencia, N. (2015). La industria química: importancia y retos. Lámpsakos, 14, 72. https://doi.org/10.21501/21454086.1562

Ninou-Collado, P. (2017). Propuesta de intervención para la enseñanza de la química desde un enfoque en contexto (trabajo de fin de máster, Universidad Internacional de La Rioja). https://reunir.unir. net/bitstream/handle/123456789/6488/ NINOU $\% 20$ COLLADO $\% 2$ C $\% 20$ PATRICIA. pdf? sequence $=1$ \&isAllowed $=y$

Ordaz González, G. J., Mostue, M. B. (2018). Los caminos hacia una enseñanza no tradicional de la química. Actualidades Investigativas en Educación, 18(2). https://doi.org/10.15517/aie.v18i2.33164

Ordenes, R., Arellano, M., Jara, R., Merino, C. (2014). Representaciones macroscópicas, submicroscópicas y simbólicas sobre la materia. Educación Química, 25(1), 46-55. https://doi. org/10.1016/S0187-893X(14)70523-3

Perera, M. (2003). A propósito de las representaciones sociales. Apuntes teóricos, trayectoria 
y actualidad. Centro de Investigaciones Psicológicas y Sociológicas. http://biblioteca. clacso.edu.ar/Cuba/cips/20130628110808/ Perera perez repr sociales.pdf

Sanchez, J. M. P. (2017). Integrated Macro-Micro-Symbolic Approach in Teaching Secondary Chemistry. Kimika, 28(2), 22-29. https://doi.org/10.26534/kimika. v28i2.22-29

Strauss, A., Corbin, J. (2002). Bases de la investigación cualitativa: técnicas y procedimientos para desarrollar la teoría fundamentada. Universidad de Antioquia.

Taber, K. S. (2013). Revisiting the chemistry triplet: Drawing upon the nature of chemical knowledge and the psychology of learning to inform chemistry education. Chemistry Education Research and Practice, 14(2), 156-168. https://doi.org/10.1039/c3rp00012e

Taber, K. S. (2019a). Foundations for Teaching Chemistry (Foundation). https://doi. org/10.4324/9781351233866

Taber, K. S. (2019b). Progressing chemistry education research as a disciplinary field. Disciplinary and Interdis- ciplinary Science Education Research, 1(1). https://doi.org/10.1186/s43031-019-0011-z

Talanquer, V. (2011). Macro, submicro, and symbolic: The many faces of the chemistry "triplet". International Journal of Science Education, 33(2), 179-195. https://doi.org/10.1080/09500690903386435

Talanquer, V. (2018a). Formación docente ¿Qué conocimiento distingue a los buenos maestros de química? Educación Química, 15(1), 52. https://doi.org/10.22201/ fq.18708404e.2004.1.66216

Talanquer, V. (2018b). Chemical rationales: another triplet for chemical thinking. International Journal of Science Education, 40(15), 1874-1890. https://doi.org/10.1080/09500693.2018.1513671

Tamayo, O., Zona, R., Loaiza, Y. (2015). El pensamiento crítico en la educación. Algunas categorías centrales en su estudio. Revista Latinoamericana de Estudios Educativos (Colombia), 11(2), 111-133.

Verdú, C. P., Chica, A. A. (2015). La investigación cualitativa: técnicas de investigación y análisis con Atlas.ti. Universidad de Alicante. http://hdl.handle.net/10045/52606 